\title{
Changes in the 21st Century: Substituting Virtual Reality Simulation Experiments for Reality Laboratories
}

\author{
Maye Aenne Rigue \\ Visayas State University- Tolosa Campus \\ miguemaeanne@gmail.com
}

\begin{abstract}
Science played an important role in our daily lives, in fact it has been a part of our curriculum and educational system. Part of it is developing scientific knowledge and science process skills through different activities conducted in the laboratory. As the years goes by, the performing of tasks in laboratories are lessened. With all the trends and improvements of technology nowadays, educational tools and technology has also improved and dominates our classroom and one of which is the usage of virtual laboratories. This journal aimed to showcase the effect of replacing actual laboratories with virtual laboratories. The author studied the results and arguments of some researchers about the usage of actual and virtual laboratories. The author concluded that choosing virtual laboratory to replace actual laboratory would be a great picture of a chaotic scenario for science education that is always aimed to instill scientific literacy to each and every student. Virtual laboratory can't replace the learning that actual laboratory can cater but it will help improve and cure misconceptions, it can be an alternative of supportive learning environment but can't replace actual laboratory.
\end{abstract}

Keywords: actual laboratory, virtual laboratory, science education, science process skills

\section{Introduction}

Science education has been a part of our curriculum for so many years. It embodies the goal needs hands on experiences with the use of science laboratories. But because of this emerging issue of the use of technology in education it was argued that it has dramatically changed the setup of learning in the science laboratory[14]. Different experiments done in the laboratories are activities that could attract students' interest towards science subjects [18]. Students are allowed to explore and conduct experiments based on science concepts they learn in the classroom. Laboratory activities are also considered activities for experiential learning that is very crucial in teaching and learning science [2][12]. It is a well-known verse that experience is the best teacher. Correlating it with experiences in the laboratory, students learn many thingsfrom factual concepts to ideas that brings out curiosity. And experience is an activity with involvement where learning and development happen [17].

Laboratories has a significant role in effective science education as well as comprehending science related laws, principles and concepts[9]. It is also argued that students develop different skills as they are engaged in different laboratory activities [7] [8] [20]. But because of the advent of technology new setup for science laboratory is currently used in different schools. It is called virtual laboratory where all the activities and experiments of the students are programmed and simulated.

\section{Virtual Laboratory}

Virtual laboratories are imitation of actual laboratories that are highly programmable that serves as simulations wherein students can move objects and perform an experiment through the use of mouse and computer monitor [11]. It is considered cheaper than the actual laboratory wherein you need to buy different materials before undergoing experiments that may include expensive chemicals and apparatuses [2]. In this type of laboratory, the students just sit in front of a computer monitor and is holding a mouse while doing the laboratory activity unlike in the actual laboratory wherein students set up all the necessary equipment before proceeding to the experiment. Here, they are only to follow the procedure based on the programmed data and there they'd be able to gather the data they needed to verify. But the data gathered by the students after the activity are not real because it is computer generated and already programmed ([14]) thus, students can predict the results and the authenticity of data is not reliable.

According to Ince et al. (2014) virtual laboratory programs are defined as programs that are adapted according to the students' and teachers' needs, and that integrate modelling, simulation and information technologies in order to create an environment of high interaction. This helps create an ideal environment that would help teaching and learning[11].

There are studies showing that virtual laboratories are as effective in achieving learning outcomes as the actual laboratory because it is viewed as a laboratory that is learner- centered and inquiry based wherein students develop higher order thinking skills [2][7] [12] [13] [14]. But Ma and Nickerson (2006) argued using the theory of situated learning that students performing laboratory activities using simulations learn how to run simulations and not science concepts. But the good thing about virtual laboratories is that it covers up the insufficiency and absence of actual laboratories [6]. It allows teachers to achieve learning objectives even with the absence of laboratory equipment. Laboratory activities needs funding 
and a school with insufficient funding may resort to virtual laboratories especially when the class number is too big [3].

In the trend of education nowadays, technology has a great impact on the learning and teaching process especially science education. And one that is in demand is virtual laboratories that has a goal of replacing the actual laboratories [11], but this paper would like to argue that virtual laboratories can't replace the quality of learning the actual laboratory caters for the students as well as the teachers. This paper will show that virtual laboratories restrict students to explore beyond their curiosity, it hinders students to develop different science process skills, lessens the total academic experience of the students and confined learning to theoretical knowledge.

\section{Restricting Students to Explore}

Learning science involves discovery learning wherein students explore in the laboratory. Students appreciate and learn science through meaningful direct and actual experiences [10]. In the laboratory they manipulate and observe things that may boost their curiosity to explore any related researches that a virtual laboratory can't cater because the setup in the virtual laboratory are already programmed and students can't manipulate and change and add variables to get an answer on what they wanted to know.

In 2018, Faulconer and Gruss pointed out that the common argument that supports actual laboratory experiences is their role in developing practical skills needed to conduct more advanced research. This is where students learn to construct their own hypothesis and explore for facts and results that may support or contradict their educated guess. Since in a virtual laboratory student only follow steps of the programmed simulations to be able to gather the desired data, they can't test their hypothesis because the programs are limited to what the learning outcome of the virtual lab activity is seeking to attain.

Science education laboratories usually let students interact with real equipment wherein during an experiment of activity they can use and manipulate to gather data and draw conclusions. According to Triona and Klahr's (2009) assumption that the difference of actual laboratories and virtual laboratories is the mode of discovery learning. It is because students are able explore and observe different experiments like reactions of chemical compounds. But according to Burkett and Smith (2016) simulated laboratories also allow students to explore and conduct laboratory experiments that are logistically impractical and not safe if done in an actual laboratory setup. But conducting this logistically impractical and unsafe laboratory activity like what has been mentioned earlier could be meaningful for the students to be motivated to conduct further studies. And unsafe laboratory activities are guided by the teachers and there are laboratory safety protocols. Confining students' laboratory activities to simulated activities to promote safety is quite reasonable but letting them observe reactions and results of experiments that may boost their interest to explore more about science is much reasonable for actual laboratories to be the utmost priority than that of virtual laboratory. As quoted by Ma and Nickerson (2006) "hands-on experience is the heart of science learning". And it shows in the study conducted by Rojko, Debevc, and Hercog (2009) that many opinions of the student who participate in the study that they learn more in actual laboratory experiments than in virtual laboratories. It is because they are actively involved in the learning process [10] [16].

\section{Hindering Laboratory Skill Development}

As mentioned earlier the purpose of creating virtual laboratories is to replace the actual laboratories [11] which is not possible because actual laboratories develop skills that a virtual laboratory can't provide. Science educators consider laboratory as a medium for students to collaborate through forming small groups in investigating phenomena and evaluating science facts [4] [20]. Thus, this only implies that laboratories create an environment where studentstudent and teacher- student relationship is built through teamwork[4][5][7] [14] which a virtual laboratory can't offer because students are interacting with computers and simulated data and not with their classmates and teacher. It is evident that collaborative skills are not developed in a virtual laboratory.

According to Unal and Ozdemir (2013), collaborative methods provide situations where participants must offer explanations, interpretations, and resolutions to problems [20]. And thus, students develop higher order thinking skills as they work in an actual laboratory due to the complexity of the activities being accomplished [4] and while dealing with this complex activity they evaluate science concepts collaboratively. There is a room for arguments and deeper discussion as they perform the activity.

Aside from higher order thinking skills, in the laboratory all types of learners are accommodated-from visual learners, aural learners and kinesthetic learners, because all types of senses are involved. Students have equal opportunities to perform in the activity, they observe, manipulate and discuss the things that is happening during a laboratory session [4].

\section{As quoted by Burkett and Smith(2016);}

"Students do not organize or set up simulated laboratory experiments, so do not build organization skills. Students do not physically operate laboratory equipment in simulated laboratories, so do not learn to use laboratory equipment. Students do not have the opportunity to practice precise motor skills and measurements in simulated laboratories."

In an actual laboratory the students organize experiments on their own. They develop organizational and procedural skills because they are the one who set up the equipment needed before proceeding to the main activity. And they are made aware that one wrong set up may result to inaccurate data that's why they became mindful of every step that they are about to do.

Laboratory is a place where there is process learning and where scientific concepts are practiced [5][10]. There is better understanding of the science process skills because 
these skills are directly catered by the laboratory activities itself [20]. While performing the activity the students don't notice that they are already developing science process skills which they could use in their specific fields of specialization. Actual laboratories also cater procedural skills where students learn how to follow systematic steps to be able to perform the activity correctly [7]. It also develops the skill on solving problems and phenomena that can be explained by scientific laws [8][10]. Its main goal is to provide activities is for laboratory experiences wherein students develop how to deduce ideas and use that for scientific reasoning as well as construct hypothesis that could be used in undergoing future research [7][8]. Students learn to explain, propose or even contradict to ideas based of scientific facts.

According to Abdulwahed and Nagy (2009), laboratories can be correlated with the fact that engineering is, in general, an applied science that requires hands-on skills and involves elements of design, problem solving, and analytical thinking. Thus, it is essential for a laboratory to develop science process skills. A laboratory that do not develop such skills may not be called a laboratory at all. In their article entitled Applying Kolb's Experiential Learning Cycle for Laboratory Education they have showed Kolb's Experiential Learning Theory (1984) as part of laboratory education. According to Kolb, as cited by Abdulwahed and Nagy 2009, learning requires that individuals first should detect, depict, or grasp knowledge, and then a phase of construction should take place to complete the learning process. Thus, this implies that laboratories construct different skills [7] [9] [20] as well as concrete knowledge. Given the fact that the virtual laboratory are just imitations of the actual laboratory the students may gather programmed data and not the real data that they may gather in an actual laboratory activity therefore students may not develop the skill of trial and error in identifying the accuracy of data in different trials[14].

\section{Lessening Academic Experience}

Active learning is a part of structuring and constructing knowledge that is supported by a learning theory called constructivism [11]. Actual laboratory is with cognitive development from leaning the concrete to abstract concepts [12]. Here students learn to deduce complex ideas through the use of laboratory experiments and they were able to construct their own learning which is meaningful for them because they are actively involved.

During a virtual laboratory activity teacher instruction and assistance is not present, lessening students peer collaboration and guidance towards achieving the concept needed to be portrayed at the end of the activity [7]. Also, the variables are fixed and limited for the students to investigate ideas [4]. Making the learning experience of the students limited to the virtual activities and simulations, thus, lessening academic experience wherein they can explore and go beyond what is thought in the classroom.

In the study of Triona and Khalr (2007), they said that when comparing physical and virtual materials, there are at least three potentially important dimensions to consider:
(1)What is being taught? Is it a process skill, such as creating controlled experiments or filling out tables of data, or is it particular facts and concepts, such as the speed of light or the structure of the carbon atom? (2) How is it being taught? Where on the broad spectrum from explicit instruction to discovery learning is it? (3) Is it hands-on or hands-off instruction?

Let's first consider the first dimension pointed out by Triona and Khalr (2007) which is "What is to be taught?" In virtual laboratory concepts can be taught but science process skills are not unlike in an actual laboratory where concepts and skills are both considered. Second, "How is it being taught?" Well, in a virtual laboratory concepts are taught through simulations and programmed software unlike in an actual laboratory there is validation and evaluation of concepts through observation and actual experimentation where students can manipulate variables of the experiments. And lastly, "Is it hands-on and hands-off instruction?" In learning science, concepts may be learned through hands-on and hands-off, but it depends upon the complexity of the concepts to be learned.

Thus, with all these dimensions to consider, if we choose virtual laboratory over actual laboratory we literally lessen the academic experience of the students. In actual laboratory all learning outcomes to be considered for students to learn not only concepts as well as skills are considered [4][5][7] [14] while virtual laboratory limits students' curiosity and confined it to theoretical knowledge only.

\section{Narrowing Students Learning to Theoretical Knowledge}

Scientific knowledge is categorized into two domains which are content or theoretical knowledge and process skills or practical knowledge. Laboratories provide exercises wherein students verify and establish scientific laws, principles and concepts through manipulating variables and witnessing results of experiments [20]. It is not only aimed to reinforce theoretical knowledge as what virtual laboratories do, but also let students discover knowledge on their own like what actual laboratories aimed [5] [8].

Ma and Nickerson (2006) distinguish two characteristics of actual laboratory from virtual laboratories:

(1) All the equipment required to perform the laboratory is physically setup; and (2) the students who perform the laboratory are physically present in the lab. Advocates argue that hands-on labs provide the students with real data and "unexpected clashes"- the disparity between theory and practical knowledge.

With this we would be able to see that actual laboratories are not only focusing to establish theoretical knowledge but also practical knowledge of the students that would be very beneficial for them in performing future researches as well as skills that would be needed in their specific fields. This characteristic of an actual laboratory is its strength compared to virtual laboratory that only narrows the learning of the students to theoretical knowledge. Students that are exposed in virtual laboratories are learning concepts and but has low development of science process skills [5][7] 
[14]. It is focused on programmed data and hinders skill development and practical knowledge.

\section{Implications}

As mentioned by Burkett and Smith (2016) laboratories are part of the local and national standards. Thus, laboratory, being one of the important learning arenas for science education, has its major role in boosting economy. In the laboratory different scientific skills are being developed [5][7]. These skills are essential for a country to produce empowered Science Technology Engineering and Mathematics (STEM) graduates that would help build the economy and bring sustainable development. Individuals that are scientific literate and is locally and globally competitive.

There are no concrete standards to compare the effectiveness of virtual and actual laboratory [7]. And also, there is no consent on the efficiency because each laboratoryis used to attain different educational outcomes and is using different instruments/methods [14]. But imagine science education without actual laboratory and we choose virtual laboratory, students will learn concepts but won't be able to apply its use in real life setting. Students tend to lack development of science process skills which are important when they pursue science related courses. And how can we produce science literate individuals that think sustainably for the environment as well as societal development if we restrain students learning to concepts only?

Actual laboratory is a good learning arena especially for science education but there are instances that some concepts are not clearly stated by the teacher leading to students' confusion. And here is where virtual laboratory should take its role, improving conceptual understanding of the students that were not able to address during the hands-on lab session [1]. It could serve as a preferable alternative or simply supportive learning environment for actual laboratories [9][12] [19] but not to replace actual laboratories [2].

\section{Conclusion}

With all the trends and improvements of technology nowadays, educational tools and technology has also improved and dominates our classroom. It has been the medium for instruction and contributes to the learning process of the students. We tend to make life of the students easier as well as engaging but we tend to forget the good things that traditional learning mode can contribute to students' learning. Virtual laboratory is one of the changes that technology brought to education. Its goal was to replace actual laboratory [11] but we forget that actual laboratory is the only place where students can learn science process skills [4]. It is where students evaluate and validate scientific principles and laws that is taught in lectures inside the classroom. Here, there is collaboration between students as well as teachers in establishing concepts and learning is not restricted to concepts only because students explore and learn.
Thus, choosing virtual laboratory to replace actual laboratory would be a great picture of a chaotic scenario for science education that is always aimed to instill scientific literacy to each and every student. How can we consider a student scientific literate if he is equipped with concepts but lacks science process skills? Virtual laboratory can't replace the learning that actual laboratory can cater but it will help improve and cure misconceptions, it can be an alternative of supportive learning environment but can't replace actual laboratory. Learning is fruitful and meaningful when students are actively involved. Let's not ruin the quality of learning science because of the advent of technology, rather improve it and not replace it.

\section{References}

[1] Abdulwahed, M. and Nagy, Z.K. (2009). Applying Kolb's experiential learning cycle for laboratory education. Journal of Engineering Education; Washington Volume 98, Issue 3 (Jul 2009): 283-294

[2] Bortnik, B., Stozhko, N., Pervukhina, I., Tchernysheva, A. and Belysheva, G. (2017). Effect of virtual analytical chemistry laboratory on enhancing student research skills and practices. Research in Learning Technology Vol. 25, 2017

[3] Breakey, K.M., Levin, D., Miller, I. and Hentges, K. (2008). The use of scenario based-learning interactive software to create custom virtual laboratory scenarios for teaching genetics.Genetics Society of America DOI: 10.1534/genetics.108.090381

[4] Burkett, V.C. and Smith, C. (2016). Simulated vs. hands-on laboratory position paper. Electronic Journal of Science Education Vol. 20, No. 9 (2016)

[5] Corter, J.E., Esche, S.K., Chassapis, C., Ma, J. and Nickerson, J.V. (2011).Process and learning outcomes from remotely-operated, simulated and hands-on student laboratories. Computers \& Education 57 (2011) 2054-2067

[6] Faour, M.A. \& Ayoubi, Z. (2018). The effect of using virtual laboratory on grade 10 students' conceptual understanding and their attitudes towards physics. Journal of Education in Science, Environment and Health (JESEH), $\quad 4(1), \quad$ 54-68. DOI:10.21891/jeseh.387482

[7] Faulconer, E.K. and Gruss A.B. (2018). A review to weigh the pros and cons of online, remote, and distance science laboratory experiences. International Review of Research in Open and Distributed Learning Volume 19, Number 2

[8] Feyzioğlu, B. (2009). An investigation of the relationship between science process skills with efficient laboratory use and science achievement in chemistry education. Journal of TURKISH SCIENCE EDUCATION Volume 6, Issue 3, December 2009

[9] Grout, I. (2017).Remote laboratories as a means to widen participation in stem education. Education Sciences 2017, 7, 85; doi: 10.3390/educsci7040085 